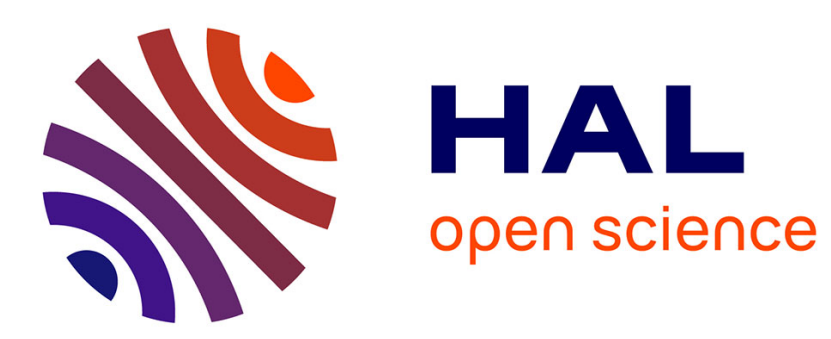

\title{
UNIVERSAL TAYLOR SERIES WITH RESPECT TO A PRESCRIBED SUBSEQUENCE
}

\author{
Augustin Mouze
}

\section{To cite this version:}

Augustin Mouze. UNIVERSAL TAYLOR SERIES WITH RESPECT TO A PRESCRIBED SUBSE-

QUENCE. 2020. hal-02877778v2

\section{HAL Id: hal-02877778 \\ https://hal.science/hal-02877778v2}

Preprint submitted on 20 Oct 2020

HAL is a multi-disciplinary open access archive for the deposit and dissemination of scientific research documents, whether they are published or not. The documents may come from teaching and research institutions in France or abroad, or from public or private research centers.
L'archive ouverte pluridisciplinaire HAL, est destinée au dépôt et à la diffusion de documents scientifiques de niveau recherche, publiés ou non, émanant des établissements d'enseignement et de recherche français ou étrangers, des laboratoires publics ou privés. 


\title{
UNIVERSAL TAYLOR SERIES WITH RESPECT TO A PRESCRIBED SUBSEQUENCE
}

\author{
A. MOUZE
}

\begin{abstract}
For a holomorphic function $f$ in the open unit disc $\mathbb{D}$ and $\zeta \in \mathbb{D}, S_{n}(f, \zeta)$ denotes the $n$-th partial sum of the Taylor development of $f$ at $\zeta$. Given an increasing sequence of positive integers $\mu=\left(\mu_{n}\right)$, we consider the classes $\mathcal{U}(\mathbb{D}, \zeta)$ (resp. $\mathcal{U}^{(\mu)}(\mathbb{D}, \zeta)$ ) of such functions $f$ such that the partial sums $\left\{S_{n}(f, \zeta): n=1,2, \ldots\right\}$ (resp. $\left\{S_{\mu_{n}}(f, \zeta): n=1,2, \ldots\right\}$ ) approximate all polynomials uniformly on the compact sets $K \subset\{z \in \mathbb{C}:|z| \geq 1\}$ with connected complement. We show that these two classes of universal Taylor series coincide if and only if $\lim \sup _{n}\left(\frac{\mu_{n+1}}{\mu_{n}}\right)<+\infty$. In the same spirit, we prove that, for $\zeta \neq 0$, we have the equality $\mathcal{U}^{(\mu)}(\mathbb{D}, \zeta)=\mathcal{U}^{(\mu)}(\mathbb{D}, 0)$ if and only if $\lim \sup _{n}\left(\frac{\mu_{n+1}}{\mu_{n}}\right)<+\infty$. Finally we deal with the case of real universal Taylor series.
\end{abstract}

\section{INTRODUCTION}

As usual $\mathbb{N}, \mathbb{Q}$ denote the sets of positive integers and rational numbers respectively. Let $\mathbb{D}:=$ $\{z \in \mathbb{C}:|z|<1\}$ be the open unit disc of the complex plane. Throughout the paper, $H(\mathbb{D})$ denotes the vector space of all holomorphic functions on $\mathbb{D}$ endowed with the topology of uniform convergence on all compact subsets of $\mathbb{D}$. Also for a compact set $K$ of $\mathbb{C}$ we denote by $A(K)$ the set of all functions which are holomorphic in the interior $K^{\circ}$ of $K$ and continuous on $K$. As usual, for a holomorphic function $f$ in the unit disc and $\zeta \in \mathbb{D}, S_{n}(f)$ or $S_{n}(f, \zeta)$ stands for the $n$-th partial sum of the Taylor development of $f$ with center at 0 or at $\zeta$ respectively. In 1996, Nestoridis proved the following result [13].

Theorem 1.1. 13] There exist Taylor series $f=\sum_{n \geq 0} a_{n} z^{n}$ such that, for every compact set $K \subset\{z \in \mathbb{C} ;|z| \geq 1\}$ with connected complement and for every function $h \in A(K)$ there exists a subsequence $\left(\lambda_{n}\right) \subset \mathbb{N}$ such that $S_{\lambda_{n}}(f)$ converges to $h$, as $n \rightarrow+\infty$, uniformly on K. will

In the sequel, such Taylor series will be called universal Taylor series and we will denote by $\mathcal{U}(\mathbb{D}, 0)$ the set of such universal Taylor series. In the same spirit, for $\zeta \in \mathbb{D}$, we can replace $S_{\lambda_{n}}(f)$ by $S_{\lambda_{n}}(f, \zeta)$ in the previous theorem to obtain the class $\mathcal{U}(\mathbb{D}, \zeta)$ of universal Taylor series with center $\zeta$. The sets $\mathcal{U}(\mathbb{D}, 0)$ or $\mathcal{U}(\mathbb{D}, \zeta)$ enjoy very strong properties. For example, these sets are $G_{\delta}$ dense subsets of $H(\mathbb{D})$ and contain, apart from 0 , a dense vector subspace of $H(\mathbb{D})$ 2, 13. This last property means that the sets $\mathcal{U}(\mathbb{D}, 0)$ and $\mathcal{U}(\mathbb{D}, \zeta)$ are algebraically generic. A very nice theorem asserts that for all $\zeta \in \mathbb{D}, \mathcal{U}(\mathbb{D}, 0)=\mathcal{U}(\mathbb{D}, \zeta)$. We refer the reader to [5] and [9]. A crucial tool for the proof is a result initiated by Gehlen, Luh and Müller [5] which asserts that every universal Taylor series actually possesses Ostrowski-gaps, in the sense of the following definition.

Definition 1.2. Let $\zeta \in \mathbb{C}$. Let $\sum_{j=0}^{+\infty} a_{j}(z-\zeta)^{j}$ be a complex power series with radius of convergence $r \in(0,+\infty)$. We say that it has Ostrowski-gaps $\left(p_{m}, q_{m}\right)$ if $\left(p_{m}\right)$ and $\left(q_{m}\right)$ are sequences of natural numbers with

(1) $p_{1}<q_{1} \leq p_{2}<q_{2} \leq \ldots$ and $\lim _{m \rightarrow+\infty} \frac{q_{m}}{p_{m}}=+\infty$,

(2) for $I=\cup_{m=1}^{\infty}\left\{p_{m}+1, \ldots, q_{m}\right\}$, we have $\lim _{j \in I}\left|a_{j}\right|^{1 / j}=0$.

The fact that every universal Taylor series possesses Ostrowski-gaps is at the core of many beautiful results (see for instance [1, 3, 5, 7, 9, 11]). Thus the combination of the existence of

2010 Mathematics Subject Classification. 30K05.

Key words and phrases. Universal Taylor series.

The author was partly supported by the grant ANR-17-CE40-0021 of the French National Research Agency ANR (project Front). 
Ostrowski-gaps with a result of Luh (see Theorem 1 of 8 and Lemma 2.3 below) allows to obtain the aforementioned equality $\mathcal{U}(\mathbb{D}, 0)=\mathcal{U}(\mathbb{D}, \zeta)$. Notice that we give a new proof of 8 , Theorem 1] in Section 2.1. Next, in order to prove the algebraic genericity of the class of universal Taylor series, the following subclass of universal series was introduced.

Definition 1.3. Let $\zeta \in \mathbb{D}$. Let $\mu=\left(\mu_{n}\right)$ be an increasing sequence of positive integers with $\mu_{n} \rightarrow+\infty$ as $n$ tends to infinity. A holomorphic function $f \in H(\mathbb{D})$ belongs to the class $\mathcal{U}^{(\mu)}(\mathbb{D}, \zeta)$ if for every compact set $K \subset\{z \in \mathbb{C} ;|z| \geq 1\}$ with connected complement and for every function $h \in A(K)$ there exists a subsequence $\left(\lambda_{n}\right) \subset \mathbb{N}$ such that $S_{\mu_{\lambda_{n}}}(f, \zeta)$ converges to $h$, as $n \rightarrow+\infty$, uniformly on $K$.

Obviously we have $\mathcal{U}^{(\mu)}(\mathbb{D}, 0) \subset \mathcal{U}(\mathbb{D}, 0)\left(\right.$ or $\left.\mathcal{U}^{(\mu)}(\mathbb{D}, \zeta) \subset \mathcal{U}(\mathbb{D}, \zeta)\right)$. In [11, as a consequence of a more general result relating to the weighted densities of subsequences along which the partial sums of universal Taylor series realize the universal approximation, the authors exhibit non-trivial subsequences $\mu$ of $\mathbb{N}$ such that $\mathcal{U}^{(\mu)}(\mathbb{D}, 0)=\mathcal{U}(\mathbb{D}, 0)$. For example, the sequences $\left(\mu_{n}\right)=\left(n^{2}\right)$, $\left(\mu_{n}\right)=\left(2^{n}\right)$ or the sequence of prime numbers satisfy this property. In [11, Section 5], it is asked to characterize the subsequences $\mu$ for which the equality $\mathcal{U}^{(\mu)}(\mathbb{D}, 0)=\mathcal{U}(\mathbb{D}, 0)$ holds. In this paper, we are going to answer this question by establishing the following result.

Theorem 1.4. Let $\zeta \in \mathbb{D}$. Let $\mu=\left(\mu_{n}\right)$ be a strictly increasing sequence of positive integers. The following assertions are equivalent:

(i) $\mathcal{U}(\mathbb{D}, \zeta)=\mathcal{U}^{(\mu)}(\mathbb{D}, \zeta)$

(ii) $\limsup _{n \rightarrow+\infty}\left(\frac{\mu_{n+1}}{\mu_{n}}\right)<+\infty$.

To prove the implication (2) $\Rightarrow$ (11) we use in an essential way the fact that all universal Taylor series possess Ostrowski-gaps. To obtain the converse implication, we employ a constructive method based on a Bernstein-Walsh type theorem given by Costakis and Tsirivas (see Theorem 2.4 below), when they studied the phenomenon of disjoint universal Taylor series [4]. As consequence of Theorem 1.4 we show the independence of the class $\mathcal{U}^{(\mu)}(\mathbb{D}, \zeta)$ with the center of expansion $\zeta$ provided that $\limsup _{n \rightarrow+\infty}\left(\frac{\mu_{n+1}}{\mu_{n}}\right)<+\infty$. This phenomenon was already noticed in specific cases in [15, 16. Furthermore, using a constructive method similar to that of the proof of Theorem 1.4 and the ideas of the new proof of [ 8 , Theorem 1$]$, we obtain the following characterization.

Theorem 1.5. Let $\zeta \in \mathbb{D}, \zeta \neq 0$. Let $\mu=\left(\mu_{n}\right)$ be a strictly increasing sequence of positive integers. The following assertions are equivalent:

(i) $\mathcal{U}^{(\mu)}(\mathbb{D}, \zeta)=\mathcal{U}^{(\mu)}(\mathbb{D}, 0)$

(ii) $\limsup _{n \rightarrow+\infty}\left(\frac{\mu_{n+1}}{\mu_{n}}\right)<+\infty$.

We refer the reader to Corollary 2.6 and Theorem 2.7. Finally in the last section we deal with the case of real universal Taylor series.

\section{Universal TAYlor SERIES VERSus Universal TAYlor SERIES With RESPECT to A PRESCRIBED SUBSEQUENCE}

2.1. Preliminary results. In this subsection we state some results that we will use for the proof of the main theorem. On one hand we are interested in the fact that all universal Taylor series possess Ostrowski-gaps. Actually a slightly more precise result holds (se [9, Theorem 9.1]).

Lemma 2.1. Let $\zeta \in \mathbb{D}$. Let $f \in \mathcal{U}(\mathbb{D}, \zeta)$. Let $K \subset \mathbb{C} \backslash \mathbb{D}$ be a compact set with connected complement and let $h \in A(K)$. Then there exist two sequences of positive integers $\left(p_{m}\right),\left(q_{m}\right)$ such that

(1) the Taylor series of $f$ at $\zeta$ has Ostrowski-gaps $\left(p_{m}, q_{m}\right)$,

(2) and $\sup _{z \in K}\left|S_{p_{m}}(f, \zeta)(z)-h(z)\right| \rightarrow 0$, as $m \rightarrow+\infty$. 
Combined Lemma 2.1 with the definition of universal Taylor series we immediately deduce the following useful lemma.

Lemma 2.2. Let $\zeta \in \mathbb{D}$. Let $f$ be in $H(\mathbb{D})$ and suppose that the Taylor series of $f$ at 0 has Ostrowski-gaps $\left(p_{m}, q_{m}\right)$. Then for every sequence $\left(r_{m}\right)$ with $p_{m}<r_{m} \leq q_{m}$ the difference between partial sums $S_{r_{m}}(f, \zeta)(z)-S_{p_{m}}(f, \zeta)(z)$ converges uniformly to zero (as $m \rightarrow+\infty$ ) on compact sets of $\mathbb{C}$.

In connection with the Ostrowski-gaps, we also have the following result ([8, Theorem 1] or [9, Lemma 9.2]). The published proof uses the Hadamard three-circle theorem. Here we give an elementary proof of the result.

Lemma 2.3. Let $f$ be in $H(\mathbb{D})$ and $\zeta_{0}, \zeta \in \mathbb{D}$. Suppose that the Taylor series of $f$ at $\zeta_{0}$ has Ostrowski-gaps $\left(p_{m}, q_{m}\right)$. Then the difference $S_{p_{k}}(f, \zeta)(z)-S_{p_{k}}\left(f, \zeta_{0}\right)(z)$ converges to zero (as $k \rightarrow+\infty)$ uniformly on compact sets of $\mathbb{D} \times \mathbb{C}(\zeta \in \mathbb{D}, z \in \mathbb{C})$.

Proof. Without loss of generality, we can suppose that $\zeta_{0}=0$ (and $\zeta \neq \zeta_{0}$ !). Let $K \subset \mathbb{C}$ and $L \subset \mathbb{D}$ be fixed compact sets. We define

$$
r=\sup _{\zeta \in L}|\zeta| \text { and } M>\max (2, \sup \{|z-\zeta|: z \in K, \zeta \in L\}) .
$$

Let us choose $0<\varepsilon<1$ such that

$$
r(1+\varepsilon)<1 \text { and } 2 \varepsilon M<1 .
$$

We write, for all $|z|<1, f(z)=\sum_{k=0}^{+\infty} a_{k} z^{k}$. Thus, for all $j \geq 0$, we have $f^{(j)}(\zeta)=j ! \sum_{k=j}^{+\infty} a_{k}\left(\begin{array}{c}k \\ j\end{array}\right) \zeta^{k-j}$. Using the equality $z^{k}=\sum_{l=0}^{k} a_{k}\left(\begin{array}{l}k \\ l\end{array}\right)(z-\zeta)^{l} \zeta^{k-l}$, we get, for all $\zeta \in L$,

$$
\begin{aligned}
S_{p_{m}}(f, \zeta)(z)-S_{p_{m}}(f)(z)= & \sum_{j=0}^{p_{m}}\left(\sum_{k=j}^{+\infty} a_{k}\left(\begin{array}{l}
k \\
j
\end{array}\right) \zeta^{k-j}\right)(z-\zeta)^{j}-\sum_{j=0}^{p_{m}}\left(\sum_{k=j}^{p_{m}} a_{k}\left(\begin{array}{l}
k \\
j
\end{array}\right) \zeta^{k-j}\right)(z-\zeta)^{j} \\
= & \sum_{j=0}^{p_{m}}\left(\sum_{k=1+p_{m}}^{q_{m}} a_{k}\left(\begin{array}{c}
k \\
j
\end{array}\right) \zeta^{k-j}\right)(z-\zeta)^{j} \\
& +\sum_{j=0}^{p_{m}}\left(\sum_{k=1+q_{m}}^{+\infty} a_{k}\left(\begin{array}{c}
k \\
j
\end{array}\right) \zeta^{k-j}\right)(z-\zeta)^{j} \\
:= & A_{1, m}(\zeta, z)+A_{2, m}(\zeta, z)
\end{aligned}
$$

Now we are going to estimate the series $A_{1, m}(\zeta, z)$ and $A_{2, m}(\zeta, z)$. On one hand, by the triangle inequality and the inequality $\left(\begin{array}{c}k \\ j\end{array}\right) \leq 2^{k}$, we get

$$
\sup _{\zeta \in L} \sup _{z \in K}\left|A_{1, m}(\zeta, z)\right| \leq \sum_{j=0}^{p_{m}}\left(\sum_{k=1+p_{m}}^{q_{m}}\left|a_{k}\right| 2^{k} r^{k-j}\right) M^{j} .
$$

Using the Ostrowski-gaps, one can find $m_{1}$ such that, for all $m \geq m_{1}$ and $1+p_{m} \leq k \leq q_{m}$, $\left|a_{k}\right|<\varepsilon^{k}$. We deduce, using the property $M>2 r$,

$$
\sup _{\zeta \in L} \sup _{z \in K}\left|A_{1, m}(\zeta, z)\right| \leq \sum_{j=0}^{p_{m}}\left(\sum_{k=1+p_{m}}^{q_{m}}(2 \varepsilon r)^{k}\right)\left(\frac{M}{r}\right)^{j} \leq \frac{1}{1-2 \varepsilon r}(2 \varepsilon M)^{1+p_{m}} .
$$

On the other hand, we need the following classical inequality

$$
\left(\begin{array}{l}
k \\
j
\end{array}\right) \leq \frac{k^{j}}{j !} \leq\left(\frac{e k}{j}\right)^{j}
$$


and the fact that, for $t<k$, the function $t \mapsto\left(\frac{e k}{t}\right)^{t}$ is increasing. It follows

$$
\sup _{\zeta \in L} \sup _{z \in K}\left|A_{2, m}(\zeta, z)\right| \leq \sum_{j=0}^{p_{m}}\left(\sum_{k=1+q_{m}}^{+\infty}\left|a_{k}\right| e^{p_{m}}\left(\frac{k}{p_{m}}\right)^{p_{m}} r^{k-j}\right) M^{j} .
$$

Since $p_{m} / q_{m} \rightarrow 0$, as $m$ tends to infinity, we have, for all $k \geq 1+q_{m}$ and $0 \leq j \leq p_{m}$,

$$
\begin{aligned}
e^{\frac{p_{m}}{k-j}} e^{\frac{p_{m}}{k-j} \log \left(\frac{k}{p_{m}}\right)} & \leq e^{\frac{p_{m}}{1+q_{m}-p_{m}}} e^{\frac{p_{m}}{k(1-j / k)} \log \left(\frac{k}{p_{m}}\right)} \\
& \leq e^{\frac{p_{m}}{1+q_{m}-p_{m}}} e^{-\frac{p_{m}}{k\left(1-\frac{p_{m}}{1+q_{m}}\right)} \log \left(\frac{p_{m}}{k}\right)} \rightarrow 1 \text { as } m \rightarrow+\infty .
\end{aligned}
$$

Therefore, since limsup $\left|a_{k}\right|^{1 / k} \leq 1$ and $p_{m} / q_{m} \rightarrow 0$, as $m$ tends to infinity, there exists a positive integer $m_{2}$ such that for all $m \geq m_{2}, k \geq 1+q_{m}$ and $0 \leq j \leq p_{m}$,

$$
\left(\frac{k e}{p_{m}}\right)^{p_{m} /(k-j)}\left|a_{k}\right|^{1 /(k-j)} r \leq(1+\varepsilon) r .
$$

Since the choice of $\varepsilon$ ensures $(1+\varepsilon) r<1$ and $M>(1+\varepsilon) r$, we deduce that for all $m \geq m_{2}$

$$
\begin{aligned}
\sup _{\zeta \in L} \sup _{z \in K}\left|A_{2, m}(\zeta, z)\right| & \leq \sum_{j=0}^{p_{m}}\left(\sum_{k=1+q_{m}}^{+\infty}(1+\varepsilon)^{k-j} r^{k-j}\right) M^{j} \\
& \leq \frac{((1+\varepsilon) r)^{1+q_{m}}}{1-(1+\varepsilon) r} \sum_{j=0}^{p_{m}}\left(\frac{M}{(1+\varepsilon) r}\right)^{j} \\
& \leq \frac{1+p_{m}}{1-(1+\varepsilon) r}\left(\frac{M}{(1+\varepsilon) r}\right)^{p_{m}}((1+\varepsilon) r)^{1+q_{m}} .
\end{aligned}
$$

Finally taking into account $p_{m} / q_{m} \rightarrow 0$ again and combining (11) with (2) and (5), we derive

$$
\sup _{\zeta \in L} \sup _{z \in K}\left|S_{p_{m}}(f, \zeta)(z)-S_{p_{m}}(f)(z)\right| \rightarrow 0, \text { as } m \rightarrow+\infty \text {. }
$$

Notice that the statements of [8, Theorem 1] or [9, Lemma 9.2] are given in the more general case where the open unit disc $\mathbb{D}$ is replaced by a simply connected domain $\Omega$ with $\Omega \subset \mathbb{C}$. Obviously the same proof does the job with easy modifications.

On the other hand, we will need a specific version of Bernstein-Walsh theorem. It is a polynomial approximation theorem which allows in some sense to control both the degree and the valuation of the polynomials. This elegant statement was given in [4]. For given sequence $\left(x_{n}\right),\left(y_{n}\right)$ of positive real numbers, the notation $y_{n}=O\left(x_{n}\right)$ means that the sequence $\left(y_{n} / x_{n}\right)$ is bounded.

Theorem 2.4. [4, Theorem 2.1] Let $\left(\sigma_{n}\right),\left(\tau_{n}\right)$ be strictly increasing sequences of positive integers, let $K \subset \mathbb{C} \backslash \mathbb{D}$ be a compact set with connected complement and let $r \in(0,1)$. If $1 \leq \tau_{n} / \sigma_{n} \rightarrow+\infty$ as $n \rightarrow+\infty$ and if $U$ is open in $\mathbb{C}$ with $K \subset U$, then there is $\theta \in(0,1)$ so that for every $h \in H(U)$ there exists a sequence of polynomials $\left(P_{n}\right)$ of the form

$$
P_{n}(z)=\sum_{k=\sigma_{n}}^{\tau_{n}} c_{n, k} z^{k}
$$

with

$$
\sup _{z \in K}\left|h(z)-P_{n}(z)\right|=O\left(\theta^{\tau_{n}}\right) \quad \text { and } \sup _{|z| \leq r}\left|P_{n}(z)\right|=O\left(\theta^{\tau_{n}}\right) .
$$

2.2. Proof of Theorem 1.4. In order to simplify the notations, we write the proof for the class $\mathcal{U}(\mathbb{D}, 0)$. The proof works along the same lines in the case of the class $\mathcal{U}(\mathbb{D}, \zeta), \zeta \in \mathbb{D}$.

Theorem 2.5. Let $\mu=\left(\mu_{n}\right)$ be a strictly increasing sequence of positive integers. The following assertions are equivalent:

(i) $\mathcal{U}(\mathbb{D}, 0)=\mathcal{U}^{(\mu)}(\mathbb{D}, 0)$ 
(ii) $\limsup _{n \rightarrow+\infty}\left(\frac{\mu_{n+1}}{\mu_{n}}\right)<+\infty$.

Proof. $\left(\underline{\text { iii })} \Rightarrow(\right.$ (i) $)$ : assume that $\limsup _{n \rightarrow+\infty}\left(\frac{\mu_{n+1}}{\mu_{n}}\right)<+\infty$. Since the inclusion $\mathcal{U}^{(\mu)}(\mathbb{D}, 0) \subset \mathcal{U}(\mathbb{D}, 0)$ is obvious, it suffices to prove $\mathcal{U}(\mathbb{D}, 0) \subset \mathcal{U}^{(\mu)}(\mathbb{D}, 0)$. Let also $f$ be in $\mathcal{U}(\mathbb{D}, 0)$. Thus according to Lemma 2.1 for all compact subset $K \subset \mathbb{C} \backslash \mathbb{D}$ with connected complement and for all $h \in A(K)$, there exists two sequences of positive integers $\left(p_{m}\right),\left(q_{m}\right)$ such that

(1) the Taylor series of $f$ at 0 has Ostrowski-gaps $\left(p_{m}, q_{m}\right)$,

(2) and $\sup _{z \in K}\left|S_{p_{m}}(f)(z)-h(z)\right| \rightarrow 0$, as $m \rightarrow+\infty$.

Since we have both

$$
\limsup _{n \rightarrow+\infty}\left(\frac{\mu_{n+1}}{\mu_{n}}\right)<+\infty \quad \text { and } \quad \frac{q_{m}}{p_{m}} \rightarrow+\infty \text {, as } m \rightarrow+\infty,
$$

there exists $N \in \mathbb{N}$, such that for all $m \geq N$ one can find $\mu_{j_{m}}$ with $p_{m} \leq \mu_{j_{m}}<q_{m}$. Hence we apply Lemma 2.2 to obtain

$$
\sup _{z \in K}\left|S_{p_{m}}(f)(z)-S_{\mu_{j_{m}}}(f)(z)\right| \rightarrow 0, \text { as } m \rightarrow+\infty .
$$

From the triangle inequality we get

$$
\sup _{z \in K}\left|S_{\mu_{j_{m}}}(f)(z)-h(z)\right| \rightarrow 0, \text { as } m \rightarrow+\infty .
$$

This implies $f \in \mathcal{U}^{(\mu)}(\mathbb{D}, 0)$.

(i) $\Rightarrow$ (iii): to do this, we assume that $\limsup _{n \rightarrow+\infty}\left(\frac{\mu_{n+1}}{\mu_{n}}\right)=+\infty$ and it suffices to exhibit an universal series $f \in \mathcal{U}(\mathbb{D}, 0)$ such that $f \notin \mathcal{U}^{(\mu)}(\mathbb{D}, 0)$. By hypothesis there exists an increasing subsequence of positive integers $\left(n_{j}\right)$ such that $\frac{\mu_{n_{j}+1}}{\mu_{n_{j}}} \rightarrow+\infty$ as $j \rightarrow+\infty$. We set, for all $j \geq 1$,

$$
u_{j}=\mu_{n_{j}}+1, \quad w_{j}=\mu_{n_{j}+1} \quad \text { and } v_{j}=\left\lfloor\sqrt{u_{j} w_{j}}\right\rfloor .
$$

Clearly there exists $N_{0} \in \mathbb{N}$ such that, for all $j \geq N_{0}, u_{j}<v_{j}<w_{j}$ and

$$
\frac{v_{j}}{u_{j}}=\frac{\left\lfloor\sqrt{u_{j} w_{j}}\right\rfloor}{u_{j}} \leq \sqrt{\frac{\mu_{n_{j}+1}}{\mu_{n_{j}}}} \rightarrow+\infty \text { as } j \rightarrow+\infty
$$

and

$$
\frac{w_{j}}{1+v_{j}}=\frac{w_{j}}{1+\left\lfloor\sqrt{u_{j} w_{j}}\right\rfloor} \leq \frac{\mu_{n_{j}+1}}{\sqrt{\left(1+\mu_{n_{j}}\right) \mu_{n_{j}+1}}} \rightarrow+\infty \text { as } j \rightarrow+\infty .
$$

Let $\left(f_{q}\right)$ be an enumeration of all the polynomials with coefficients in $\mathbb{Q}+i \mathbb{Q}$. Let $\left(K_{m}\right)$ be a sequence of compact sets with connected complement and $K_{m} \cap \mathbb{D}=\emptyset$ for every $m \in \mathbb{N}$ such that for every compact set $K \subset \mathbb{C} \backslash \mathbb{D}$ with connected complement there exists $n \in \mathbb{N}$ such that $K \subset K_{n}$ (see [13, Lemma 2.1]). We consider an enumeration $\left(K_{m_{s}}, f_{q_{s}}\right), s=1,2, \ldots$, of all couples $\left(K_{m}, f_{q}\right)$, $m, q=1,2, \ldots$ Let also $\left(r_{l}\right)$ be an increasing sequence of real numbers with $0<r_{l}<1$ and $r_{l} \rightarrow 1$, as $l \rightarrow+\infty$. We fix $z_{0} \in \mathbb{C} \backslash \mathbb{D}$. For all $l \geq 1$, we set $\tilde{K}_{l}=K_{l} \cup\left\{z_{0}\right\}$ and note that $\tilde{K}_{l}$ is a compact set with connected complement and $\tilde{K}_{l} \subset \mathbb{C} \backslash \mathbb{D}$. First we deal with $\tilde{K}_{m_{1}}, f_{q_{1}}$ and $r_{1}$. By applying Theorem 2.4, we find $j_{1} \in \mathbb{N}$ and polynomials

$$
P_{1}(z)=\sum_{k=u_{j_{1}}}^{v_{j_{1}}} c_{j_{1}, k} z^{k}, \quad \tilde{P}_{1}(z)=\sum_{k=v_{j_{1}}+1}^{w_{j_{1}}} c_{j_{1}, k} z^{k}
$$

such that

$$
\sup _{z \in \tilde{K}_{m_{1}}}\left|P_{1}(z)-f_{q_{1}}(z)\right|<\frac{1}{2^{2}}, \quad \sup _{|z| \leq r_{1}}\left|P_{1}(z)\right| \leq \frac{1}{2^{2}}
$$


and

$$
\sup _{z \in \tilde{K}_{m_{1}}}\left|\tilde{P}_{1}(z)+f_{q_{1}}(z)\right|<\frac{1}{2^{2}}, \quad \sup _{|z| \leq r_{1}}\left|\tilde{P}_{1}(z)\right| \leq \frac{1}{2^{2}} .
$$

Observe that we have, by the triangle inequality,

$$
\begin{aligned}
\sup _{z \in \tilde{K}_{m_{1}}}\left|P_{1}(z)+\tilde{P}_{1}(z)\right| & \leq \sup _{z \in \tilde{K}_{m_{1}}}\left|P_{1}(z)-f_{q_{1}}(z)\right|+\sup _{z \in \tilde{K}_{m_{1}}}\left|\tilde{P}_{1}(z)+f_{q_{1}}(z)\right| \\
& <\frac{2}{2^{2}} .
\end{aligned}
$$

Further we find $j_{2} \in \mathbb{N}$ with $j_{2}>j_{1}$ and polynomials

$$
P_{2}(z)=\sum_{k=u_{j_{2}}}^{v_{j_{2}}} c_{j_{2}, k} z^{k}, \quad \tilde{P}_{2}(z)=\sum_{k=v_{j_{2}}+1}^{w_{j_{2}}} c_{j_{2}, k} z^{k}
$$

such that

$$
\sup _{z \in \tilde{K}_{m_{2}}}\left|P_{1}(z)+\tilde{P}_{1}(z)+P_{2}(z)-f_{q_{2}}(z)\right|<\frac{1}{3^{2}}, \quad \sup _{|z| \leq r_{2}}\left|P_{2}(z)\right| \leq \frac{1}{3^{2}}
$$

and

$$
\sup _{z \in \tilde{K}_{m_{2}}}\left|\tilde{P}_{2}(z)+f_{q_{2}}(z)\right|<\frac{1}{2^{2}}, \quad \sup _{|z| \leq r_{2}}\left|\tilde{P}_{1}(z)\right| \leq \frac{1}{3^{2}} .
$$

Observe that we have, by the triangle inequality,

$$
\begin{aligned}
\sup _{z \in \tilde{K}_{m_{2}}}\left|\sum_{i=1}^{2}\left(P_{i}(z)+\tilde{P}_{i}(z)\right)\right| & \leq \sup _{z \in \tilde{K}_{m_{2}}}\left|P_{1}(z)+\tilde{P}_{1}(z)+P_{2}(z)-f_{q_{2}}(z)\right|+\sup _{z \in \tilde{K}_{m_{2}}}\left|\tilde{P}_{2}(z)+f_{q_{2}}(z)\right| \\
& <\frac{2}{3^{2}} .
\end{aligned}
$$

We argue by induction. Suppose that for a natural number $s \geq 2$ we have already defined integers

$$
j_{1}<j_{2}<\cdots<j_{s}
$$

and polynomials $P_{i}, \tilde{P}_{i}, i=1, \ldots, s$ such that

$$
P_{i}(z)=\sum_{k=u_{j_{i}}}^{v_{j_{i}}} c_{j_{i}, k} z^{k}, \quad \tilde{P}_{i}(z)=\sum_{k=v_{j_{i}}+1}^{w_{j_{i}}} c_{j_{i}, k} z^{k}
$$

with

$$
\begin{gathered}
\sup _{z \in \tilde{K}_{m_{s}}}\left|\sum_{i=1}^{s-1} P_{i}(z)+P_{s}(z)-f_{q_{s}}(z)\right|<\frac{1}{(s+1)^{2}}, \quad \sup _{|z| \leq r_{s}}\left|P_{s}(z)\right| \leq \frac{1}{(s+1)^{2}} \\
\quad \sup _{z \in \tilde{K}_{m_{s}}}\left|\tilde{P}_{s}(z)+f_{q_{s}}(z)\right|<\frac{1}{(s+1)^{2}}, \quad \sup _{|z| \leq r_{s}}\left|\tilde{P}_{s}(z)\right| \leq \frac{1}{(s+1)^{2}} .
\end{gathered}
$$

and

$$
\sup _{z \in \tilde{K}_{m_{s}}}\left|\sum_{i=1}^{s}\left(P_{i}(z)+\tilde{P}_{i}(z)\right)\right|<\frac{2}{(s+1)^{2}} .
$$

Using Theorem 2.4, we find $j_{s+1} \in \mathbb{N}$ with $j_{s+1}>j_{s}$ and polynomials

$$
P_{s+1}(z)=\sum_{k=u_{j_{s+1}}}^{v_{j_{s+1}}} c_{j_{s+1}, k} z^{k}, \quad \tilde{P}_{s+1}(z)=\sum_{k=v_{j_{s+1}}+1}^{w_{j_{s+1}}} c_{j_{s+1}, k} z^{k}
$$

such that

$$
\sup _{z \in \tilde{K}_{m_{s+1}}}\left|\sum_{i=1}^{s} P_{i}(z)+P_{s+1}(z)-f_{q_{s+1}}(z)\right|<\frac{1}{(s+2)^{2}}, \quad \sup _{|z| \leq r_{s+1}}\left|P_{s+1}(z)\right| \leq \frac{1}{(s+2)^{2}}
$$


and

$$
\sup _{z \in \tilde{K}_{m_{s+1}}}\left|\tilde{P}_{s+1}(z)+f_{q_{s+1}}(z)\right|<\frac{1}{(s+2)^{2}}, \quad \sup _{|z| \leq r_{s+1}}\left|\tilde{P}_{s+1}(z)\right| \leq \frac{1}{(s+2)^{2}} .
$$

Thus from the triangle inequality we get

$$
\begin{aligned}
\sup _{z \in \tilde{K}_{m_{s+1}}\left|\sum_{i=1}^{s+1}\left(P_{i}(z)+\tilde{P}_{i}(z)\right)\right| \leq} & \sup _{z \in \tilde{K}_{m_{s+1}} \mid}\left|\sum_{i=1}^{s}\left(P_{i}(z)+\tilde{P}_{i}(z)\right)+P_{s+1}(z)-f_{q_{s+1}}(z)\right| \\
& +\sup _{z \in \tilde{K}_{m_{s+1}}}\left|\tilde{P}_{s+1}(z)+f_{q_{s+1}}(z)\right| \\
& <\frac{2}{(s+1)^{2}} .
\end{aligned}
$$

The induction is valid. Finally we set

$$
f(z)=\sum_{i \geq 1}\left(P_{i}(z)+\tilde{P}_{i}(z)\right)
$$

Thanks to the second inequalities of $(8)$ and (9) this series converges on all compact subsets of $\mathbb{D}$. So $f \in H(\mathbb{D})$. The first inequality of (8) ensures that $f \in \mathcal{U}(\mathbb{D}, 0)$. Indeed, let $K \subset \mathbb{C} \backslash \mathbb{D}$ be a compact set with connected complement and $h \in A(K)$. Set $\varepsilon>0$ and $s_{0} \in \mathbb{N}$ with $1 /\left(s_{0}+1\right)^{2}<\varepsilon / 2$. By hypothesis, one can find a positive integer $p>s_{0}$ such that $K \subset K_{m_{p}}$ and $\sup _{K}\left|h-f_{q_{p}}\right|<\varepsilon / 2$. Thus from (8) we get

$$
\sup _{z \in K}\left|S_{v_{j_{p}}}(f)(z)-h(z)\right| \leq \sup _{z \in K_{m_{p}}}\left|S_{v_{j_{p}}}(f)(z)-f_{q_{p}}(z)\right|+\sup _{z \in K_{m_{p}}}\left|h(z)-f_{q_{p}}(z)\right|<\frac{1}{(p+1)^{2}}+\frac{\varepsilon}{2}<\varepsilon .
$$

Moreover observe that the property (6) implies that

$$
\left\{\mu_{n} ; n \geq n_{j_{1}}+1\right\} \cap\left(\cup_{s \geq 1}\left\{u_{j_{s}}, u_{j_{s}}+1, \ldots, w_{j_{s}}-1\right\}\right)=\emptyset .
$$

Therefore the equation (10) guarantees that, for all $n \in \mathbb{N}$,

$$
\left|S_{\mu_{n}}(f)\left(z_{0}\right)\right| \leq 2 \sum_{s \geq 1} \frac{1}{(s+1)^{2}}=\frac{\pi^{2}}{3}-2 .
$$

From this last inequality we easily deduce that $f \notin \mathcal{U}^{(\mu)}(\mathbb{D}, 0)$.

2.3. Universal Taylor series and center independence. Let us recall that the classes $\mathcal{U}(\mathbb{D}, 0)$ and $\mathcal{U}(\mathbb{D}, \zeta)$ coincide for all $\zeta \in \mathbb{D}[5$, 9]. The proof is based on Lemma 2.1, Lemma 2.2 and Lemma 2.3 which asserts that if a Taylor series $f$ has Ostrowski-gaps $\left(p_{m}, q_{m}\right)$ then the difference $S_{p_{m}}(f, \zeta)(z)-S_{p_{m}}(f, 0)(z)$ converges to zero (as $\left.m \rightarrow+\infty\right)$ uniformly on compact sets of $\mathbb{D} \times \mathbb{C}$ $(\zeta \in \mathbb{D}, z \in \mathbb{C})$. But if you can choose $q_{m} \in \mu$, there is no evidence that you can choose $p_{m} \in \mu$. Thus it is not clear that we have $\mathcal{U}^{(\mu)}(\mathbb{D}, 0)=\mathcal{U}^{(\mu)}(\mathbb{D}, \zeta)$. Nevertheless Theorem 1.4 immediately leads to the following corollary.

Corollary 2.6. Let $\zeta \in \mathbb{D}$. Let $\mu=\left(\mu_{n}\right)$ be a strictly increasing sequence of positive integers with $\limsup _{n \rightarrow+\infty}\left(\frac{\mu_{n+1}}{\mu_{n}}\right)<+\infty$. Then we have $\mathcal{U}^{(\mu)}(\mathbb{D}, \zeta)=\mathcal{U}^{(\mu)}(\mathbb{D}, 0)$.

Proof. We know that, for all $\zeta \in \mathbb{D}, \mathcal{U}(\mathbb{D}, \zeta)=\mathcal{U}(\mathbb{D}, 0)$ [5, 9]. Assume that $\limsup _{n \rightarrow+\infty}\left(\frac{\mu_{n+1}}{\mu_{n}}\right)<$ $+\infty$. Hence Theorem 1.4 ensures that $\mathcal{U}^{(\mu)}(\mathbb{D}, \zeta)=\mathcal{U}(\mathbb{D}, \zeta)$ and $\mathcal{U}^{(\mu)}(\mathbb{D}, 0)=\mathcal{U}(\mathbb{D}, 0)$. We get $\mathcal{U}^{(\mu)}(\mathbb{D}, \zeta)=\mathcal{U}^{(\mu)}(\mathbb{D}, 0)$.

Corollary 2.6 covers all the known examples of sequences $\mu$ such that $\mathcal{U}^{(\mu)}(\mathbb{D}, \zeta)=\mathcal{U}^{(\mu)}(\mathbb{D}, 0)$ [15, 16]. Moreover Corollary 2.6 is optimal in the following sense.

Theorem 2.7. Let $\zeta \in \mathbb{D}, \zeta \neq 0$. Let $\mu=\left(\mu_{n}\right)$ be a strictly increasing sequence of positive integers with $\limsup _{n \rightarrow+\infty}\left(\frac{\mu_{n+1}}{\mu_{n}}\right)=+\infty$. Then we have $\mathcal{U}^{(\mu)}(\mathbb{D}, \zeta) \neq \mathcal{U}^{(\mu)}(\mathbb{D}, 0)$. 
Proof. Let $\zeta \in \mathbb{D}, \zeta \neq 0$. We are going to build an universal series $f \in \mathcal{U}^{(\mu)}(\mathbb{D}, 0)$ such that $f \notin \mathcal{U}^{(\mu)}(\mathbb{D}, \zeta)$. Let us consider $z_{0}$ with $\left|z_{0}\right|=1$ such that $\left|z_{0}-\zeta\right|=d(\zeta, \partial \mathbb{D})$, the distance between $\zeta$ and the unit circle. By hypothesis there exists an increasing sequence of integers $\left(n_{j}\right)$ such that

$$
\frac{\mu_{n_{j}+1}}{\mu_{n_{j}}} \rightarrow+\infty, \text { as } j \rightarrow+\infty \text {. }
$$

We set, for all $j \geq 1$,

$$
u_{j}=\mu_{n_{j}}+1, \quad w_{j}=\mu_{n_{j}+1} \quad \text { and } v_{j}=\left\lfloor\sqrt{u_{j} w_{j}}\right\rfloor .
$$

As in the proof of Theorem 2.5, we have for all $j$ large enough $u_{j}<v_{j}<w_{j}$ and

$$
\frac{v_{j}}{u_{j}} \rightarrow+\infty \text { and } \frac{w_{j}}{1+v_{j}} \rightarrow+\infty \text { as } j \rightarrow+\infty \text {. }
$$

Let us also consider an enumeration $\left(K_{m_{s}}, f_{q_{s}}\right), s=1,2, \ldots$, of all couples $\left(K_{m}, f_{q}\right), m, q=1,2, \ldots$, where $\left(f_{q}\right)$ is an enumeration of all the polynomials with coefficients in $\mathbb{Q}+i \mathbb{Q}$ and $\left(K_{m}\right)$ is a sequence of compact sets with connected complement and $K_{m} \cap \mathbb{D}=\emptyset$ such that for every compact set $K \subset \mathbb{C} \backslash \mathbb{D}$ with connected complement there exists $n \in \mathbb{N}$ such that $K \subset K_{n}$. Let also $\left(r_{l}\right)$ be an increasing sequence of real numbers with $0<r_{l}<1$ and $r_{l} \rightarrow 1$, as $l \rightarrow+\infty$. For all $l \geq 1$, we set $\tilde{K}_{l}=K_{l} \cup\left\{z_{0}\right\}$. Observe that $\tilde{K}_{l}$ is a compact set with connected complement and $\tilde{K}_{l} \subset \mathbb{C} \backslash \mathbb{D}$. First we deal with $\tilde{K}_{m_{1}}, f_{q_{1}}$ and $r_{1}$. By applying Theorem 2.4, we find $j_{1} \in \mathbb{N}$ and polynomial

$$
P_{1}(z)=\sum_{k=1+v_{j_{1}}}^{w_{j_{1}}} a_{k} z^{k}
$$

such that, for all $j \geq j_{1}$,

$$
2\left|z_{0}^{j}-\left(z_{0}-\zeta\right)^{j}\right|=2\left(1-(1-|\zeta|)^{j}\right)>1
$$

and

$$
\begin{gathered}
\left|f_{q_{1}}\left(z_{0}\right)\right| \leq w_{j_{1}}, \\
\sup _{\tilde{K}_{m_{1}}}\left|P_{1}(z)-f_{q_{1}}(z)\right|<\frac{1}{2} \text { and } \sup _{|z| \leq r_{1}}\left|P_{1}(z)\right|<\frac{1}{2} .
\end{gathered}
$$

By the triangle inequality we have

$$
\left|P_{1}\left(z_{0}\right)\right| \leq\left|P_{1}\left(z_{0}\right)-f_{q_{1}}\left(z_{0}\right)\right|+\left|f_{q_{1}}\left(z_{0}\right)\right| \leq \frac{1}{2}+w_{j_{1}} \leq 1+w_{j_{1}} .
$$

We define

$$
a_{1+w_{j_{1}}}=-\frac{P_{1}\left(z_{0}\right)}{z_{0}^{1+w_{j_{1}}}-\left(z_{0}-\zeta\right)^{1+w_{j_{1}}}} .
$$

Thanks to (13) and (14), we have

$$
\left|a_{1+w_{j_{1}}}\right| \leq 2\left(1+w_{j_{1}}\right) .
$$

Then we set

$$
R_{1}(z)=a_{1+w_{j_{1}}} z^{1+w_{j_{1}}} .
$$

By induction we construct an increasing sequence of integers $\left(j_{l}\right)$, two sequences of polynomials $\left(P_{l}\right)$ and $\left(R_{l}\right)$ such that, for all $l \geq 2$,

$$
\begin{gathered}
\frac{\mu_{n_{j_{l}}}}{1+\mu_{n_{j_{l}-1}}} \rightarrow+\infty, \text { as } l \rightarrow+\infty \\
\left|f_{q_{l}}\left(z_{0}\right)\right| \leq w_{j_{l}}, \\
P_{l}(z)=\sum_{k=1+v_{j_{l}}}^{w_{j_{l}}} a_{k} z^{k}, \quad R_{l}(z)=a_{1+w_{j_{l}}} z^{1+w_{j_{l}}}
\end{gathered}
$$


with

$$
\sup _{\tilde{K}_{m_{l}}}\left|P_{l}(z)+\sum_{i=1}^{l-1}\left(P_{i}(z)+R_{i}(z)\right)-f_{q_{l}}(z)\right|<\frac{1}{2^{l}} \text { and } \sup _{|z| \leq r_{l}}\left|P_{l}(z)\right|<\frac{1}{2^{l}},
$$

and

$$
a_{1+w_{j_{l}}}=-\frac{P_{l}\left(z_{0}\right)+\sum_{i=1}^{l-1}\left(P_{i}\left(z_{0}\right)+R_{i}\left(z_{0}\right)\right)}{z_{0}^{1+w_{j_{l}}}-\left(z_{0}-\zeta\right)^{1+w_{j_{l}}}} .
$$

By the triangle inequality we have

$$
\left|P_{l}\left(z_{0}\right)\right| \leq \frac{1}{2^{l}}+w_{j_{l}}+\leq 1+w_{j_{l}}
$$

and, taking into account (13), we deduce

$$
\left|a_{1+w_{j_{l}}}\right| \leq 2\left(1+w_{j_{l}}\right) .
$$

We also define, for all $l \geq 2$,

$$
a_{k}=0 \text { for } k=2+w_{j_{l-1}}, \ldots, v_{j_{l}} .
$$

By construction we get, for all $l \geq 1$, using (18),

$$
\begin{aligned}
\sum_{j=0}^{w_{j_{l}}}\left(\sum_{k=j}^{1+w_{j_{l}}} a_{k}\left(\begin{array}{c}
k \\
j
\end{array}\right) \zeta^{k-j}\right)\left(z_{0}-\zeta\right)^{j}= & \sum_{j=0}^{w_{j_{l}}}\left(\sum_{k=j}^{w_{j_{l}}} a_{k}\left(\begin{array}{c}
k \\
j
\end{array}\right) \zeta^{k-j}\right)\left(z_{0}-\zeta\right)^{j} \\
& +a_{1+w_{j_{l}}} \sum_{j=0}^{w_{j_{l}}}\left(\begin{array}{c}
1+w_{j_{l}} \\
j
\end{array}\right) \zeta^{1+w_{j_{l}}-j}\left(z_{0}-\zeta\right)^{j} \\
= & \sum_{i=1}^{l-1}\left(P_{i}\left(z_{0}\right)+R_{i}\left(z_{0}\right)\right)+P_{l}\left(z_{0}\right) \\
& +a_{1+w_{j_{l}}}\left(z_{0}^{1+w_{j_{l}}}-\left(z_{0}-\zeta\right)^{1+w_{j_{l}}}\right) \\
= & 0 .
\end{aligned}
$$

Now we consider

$$
f(z)=\sum_{i=1}^{+\infty}\left(P_{i}(z)+R_{i}(z)\right):=\sum_{k \geq 0} a_{k} z^{k} .
$$

Thanks to the estimates (17) and (19) the series $f$ belongs to $H(\mathbb{D})$. The equations (16) and (17) ensure that, for all $l \geq 1$,

$$
\sup _{z \in K_{m_{l}}}\left|S_{w_{j_{l}}}(f)(z)-f_{q_{l}}(z)\right|<\frac{1}{2^{l}} \text {. }
$$

Since, for all $l \geq 1, w_{j_{l}}=\mu_{1+n_{j_{l}}}$, we deduce that $f$ belongs to $\mathcal{U}^{(\mu)}(\mathbb{D}, 0)$. To finish the proof, we are going to prove that $f \notin \mathcal{U}^{(\mu)}(\mathbb{D}, \zeta)$. Notice that we have

$$
S_{m}(f, \zeta)\left(z_{0}\right)=\sum_{j=0}^{m}\left(\sum_{k=j}^{+\infty} a_{k}\left(\begin{array}{l}
k \\
j
\end{array}\right) \zeta^{k-j}\right)\left(z_{0}-\zeta\right)^{j} .
$$

First we deal with the case $m=w_{j_{l}}$. We have, thanks to the properties (20) and (21),

$$
S_{w_{j_{l}}}(f, \zeta)\left(z_{0}\right)=\sum_{j=0}^{w_{j_{l}}}\left(\sum_{k=1+v_{j_{l+1}}}^{+\infty} a_{k}\left(\begin{array}{c}
k \\
j
\end{array}\right) \zeta^{k-j}\right)\left(z_{0}-\zeta\right)^{j} .
$$

Using the equation (15), we argue as in the estimates (3), (4) and (5) of the proof of Lemma 2.3 to obtain

$$
S_{w_{j_{l}}}(f, \zeta)\left(z_{0}\right) \rightarrow 0, \text { as } l \rightarrow+\infty
$$


Now, for all $l \geq 1$ and for all positive integer $m \in\left[1+w_{j_{l}}, u_{j_{l+1}}\right]$, we can write, thanks to (20) and (21) again,

$$
\begin{aligned}
S_{m}(f, \zeta)\left(z_{0}\right)= & \sum_{j=0}^{w_{j_{l}}}\left(\sum_{k=1+v_{j_{l}+1}}^{+\infty} a_{k}\left(\begin{array}{c}
k \\
j
\end{array}\right) \zeta^{k-j}\right)\left(z_{0}-\zeta\right)^{j} \\
& +\sum_{j=1+w_{j_{l}}}^{m}\left(\sum_{k=j}^{+\infty} a_{k}\left(\begin{array}{c}
k \\
j
\end{array}\right) \zeta^{k-j}\right)\left(z_{0}-\zeta\right)^{j} \\
= & \sum_{j=0}^{w_{j_{l}}}\left(\sum_{k=1+v_{j_{l+1}}}^{+\infty} a_{k}\left(\begin{array}{c}
k \\
j
\end{array}\right) \zeta^{k-j}\right)\left(z_{0}-\zeta\right)^{j} \\
& +a_{1+w_{j_{l}}}\left(z_{0}-\zeta\right)^{1+w_{j_{l}}}+\sum_{j=1+w_{j_{l}}}^{m}\left(\sum_{k=1+v_{j_{l+1}}}^{+\infty} a_{k}\left(\begin{array}{c}
k \\
j
\end{array}\right) \zeta^{k-j}\right)\left(z_{0}-\zeta\right)^{j} \\
:= & T_{1, l}\left(z_{0}\right)+a_{1+\mu_{n_{j_{l}}+1}}\left(z_{0}-\zeta\right)^{1+\mu_{j_{l}+1}}+T_{2, l}\left(z_{0}\right) .
\end{aligned}
$$

By (19) we get $\left|a_{1+w_{j_{l}}}\left(z_{0}-\zeta\right)^{1+w_{j_{l}}}\right| \rightarrow 0$, as $l$ tends to infinity. Again inspired by the estimates (31), (44) and (51) of the proof of Lemma 2.3 we get, for $i=1,2, T_{i, l}\left(z_{0}\right) \rightarrow 0$, as $l$ tends to infinity (let us recall that $m \in\left[1+\mu_{j_{l}+1}, u_{j_{l+1}}\right]$ and $\left.\left(1+v_{j_{l+1}}\right) / u_{j_{l+1}} \rightarrow+\infty\right)$. In summary we have, for all positive integer $m \in\left[w_{j_{l}}, u_{j_{l+1}}\right]$,

$$
S_{m}(f, \zeta)\left(z_{0}\right) \rightarrow 0, \text { as } l \rightarrow+\infty .
$$

By construction, we have

$$
\left\{\mu_{n} ; n \in \mathbb{N}\right\} \bigcap\left(\bigcup_{l \geq 1}\left(\left[1+u_{j_{l+1}}, w_{j_{l+1}}-1\right] \cap \mathbb{N}\right)\right)=\emptyset .
$$

From (24) and (25), we get

$$
S_{\mu_{n}}(f, \zeta)\left(z_{0}\right) \rightarrow 0, \text { as } n \rightarrow+\infty .
$$

It follows that $f \notin \mathcal{U}^{(\mu)}(\mathbb{D}, \zeta)$. This finishes the proof.

Example 2.8. Let $\zeta \in \mathbb{D}, \zeta \neq 0$. We can apply Theorem 2.7 with the sequence $\left(\mu_{n}\right)=(n !)$. Therefore we obtain $\mathcal{U}^{((n !))}(\mathbb{D}, 0) \neq \mathcal{U}^{((n !))}(\mathbb{D}, \zeta)$.

Remarks 2.9. (1) The combination of Corollary 2.6 with Theorem 2.7 gives Theorem 1.5,

(2) Notice also that Theorem 1.4, Corollary 2.6 and Theorem 2.7 remain valid for the classes of universal Taylor series $\mathcal{U}(\Omega, \zeta)$ and $\mathcal{U}^{(\mu)}(\Omega, \zeta)$ where you replace the unit disc $\mathbb{D}$ by a simply connected domain $\Omega$, with $\Omega \subset \mathbb{C}$ and $\Omega \neq \mathbb{C}$, and $\zeta \in \Omega$, provided that the universal Taylor series possess Ostrowski-gaps (see [9, 12]). To see this, it suffices to note that Lemma 2.1. Lemma 2.2. Lemma 2.3 and Theorem 2.4 remain valid in this context. Thus the proofs work along the same lines.

\section{The ReAl CASE}

As far as we know the first example of universal series was introduced by Fekete [14] which showed that there exists a real formal power series $\sum_{n \geq 1} a_{n} x^{n}$ satisfying the following universal property: for every continuous function $g$ on $[-1,1]$ with $g(0)=0$ there exists an increasing sequence $\left(\lambda_{n}\right)$ of positive integers such that

$$
\sup _{x \in[-1,1]}\left|\sum_{k=1}^{\lambda_{n}} a_{k} x^{k}-g(x)\right| \rightarrow 0, \text { as } n \rightarrow+\infty .
$$

Further combining this result with Borel's theorem we obtain $C^{\infty}$ functions vanishing at 0 whose partial sums of its Taylor series with center 0 approximate every continuous function vanishing at 0 locally uniformly in $\mathbb{R}[6]$. We denote by $C_{0}^{\infty}(\mathbb{R})$ the space of infinitely differentiable function on $\mathbb{R}$ vanishing at 0 . 
Definition 3.1. Let $\mu=\left(\mu_{n}\right)$ be an increasing sequence of positive integers with $\mu_{n} \rightarrow+\infty$ as $n$ tends to infinity. A function $f \in C_{0}^{\infty}(\mathbb{R})$ belongs to the class $\mathcal{U}^{(\mu)}$ of universal functions with respect to $\mu$ if for every compact set $K \subset \mathbb{R}$ and every continuous functions $h: \mathbb{R} \rightarrow \mathbb{R}$ with $h(0)=0$, there exists an increasing sequence $\left(\lambda_{n}\right)$ of positive integers such that

$$
\sup _{x \in K}\left|S_{\lambda_{n}}(f)(x)-h(x)\right| \rightarrow 0, \text { as } n \rightarrow+\infty .
$$

For $\mu=\mathbb{N}$, we will denote $\mathcal{U}^{(\mu)}$ by $\mathcal{U}$.

In this context, the analogue of Theorem 1.4 states as follows.

Theorem 3.2. Let $\mu=\left(\mu_{n}\right)$ be a strictly increasing sequence of positive integers. The following assertions are equivalent:

(1) $\mathcal{U}=\mathcal{U}^{(\mu)}$

(2) $\limsup _{n \rightarrow+\infty}\left(\frac{\mu_{n+1}}{\mu_{n}}\right)<+\infty$.

To prove this result, we need the following results that are the $C^{\infty}$ versions of Lemma 2.1 and Theorem 2.4 respectively.

Lemma 3.3. [11, Proposition 4.4] Let $f \in \mathcal{U}$. Let $h: \mathbb{R} \rightarrow \mathbb{R}$ be a continuous function, with $h(0)=0$. There exist two sequences of natural numbers $\left(\lambda_{n}\right),\left(\mu_{n}\right)$ such that

(1) the Taylor series of $f$ around zero has Ostrowski-gaps $\left(\lambda_{n}, \mu_{n}\right)$,

(2) $S_{\lambda_{n}}(f) \rightarrow h$, uniformly on each compact subset of $\mathbb{R}$ as $n \rightarrow+\infty$.

Lemma 3.4. [10, Lemma 3.2] Let $\left(l_{n}\right)$ and $\left(m_{n}\right)$ be two strictly increasing sequences of positive integers such that $l_{n} \leq m_{n}$ and $\frac{m_{n}}{l_{n}} \rightarrow+\infty$ as $n \rightarrow+\infty$. Let $A>0$. For every continuous function $h: \mathbb{R} \rightarrow \mathbb{R}$, with $h(0)=0$, there exists a sequence $\left(P_{n}\right)$ of real polynomials of the form $P_{n}(x)=\sum_{k=l_{n}}^{m_{n}} c_{n, k} x^{k}$, such that

$$
\sup _{x \in[-A, A]}\left|P_{n}(x)-h(x)\right| \rightarrow 0 \text {, as } n \rightarrow+\infty \text {. }
$$

Sketch of the proof of Theorem 3.2.

(2) $\Rightarrow$ (11): thanks to Lemma 3.3. it suffices to mimic the proof (iii) $\Rightarrow$ (ii) of Theorem 2.5.

(11) $\Rightarrow$ (2): we argue as in the proof of (i) $\Rightarrow$ (iii) of Theorem 2.5. We define the same sequences $\left(u_{j}\right),\left(v_{j}\right),\left(w_{j}\right)$ and we denote by $\left(f_{j}\right)$ an enumeration of all the polynomials with coefficients in $\mathbb{Q}$. Using Lemma 3.4, we construct step by step an increasing sequence of positive integers $\left(j_{s}\right)$ and polynomials and polynomials $P_{i}, \tilde{P}_{i}, i=1, \ldots, s$ such that

$$
P_{i}(x)=\sum_{k=u_{j_{i}}}^{v_{j_{i}}} c_{j_{i}, k} x^{k}, \quad \tilde{P}_{i}(x)=\sum_{k=v_{j_{i}}+1}^{w_{j_{i}}} c_{j_{i}, k} x^{k}
$$

with

$$
\sup _{x \in[-s, s]}\left|\sum_{i=1}^{s-1} P_{i}(x)+P_{s}(x)-f_{s}(x)\right|<\frac{1}{(s+1)^{2}}
$$

$$
\sup _{x \in[-s, s]}\left|\tilde{P}_{s}(x)+f_{s}(x)\right|<\frac{1}{(s+1)^{2}} .
$$


We get by the triangle inequality

$$
\begin{aligned}
\sup _{x \in[-s, s]}\left|\sum_{i=1}^{s}\left(P_{i}(x)+\tilde{P}_{i}(x)\right)\right| \leq & \sup _{\substack{x \in[-s, s]\\
}}\left|\sum_{i=1}^{s}\left(P_{i}(x)+\tilde{P}_{i}(x)\right)+P_{s}(x)-f_{s}(x)\right| \\
& \quad \sup _{x \in[-s, s]}\left|\tilde{P}_{s}(x)+f_{s}(x)\right| \\
< & \frac{2}{(s+1)^{2}}
\end{aligned}
$$

Finally let us consider the formal power series

$$
\hat{f}(x)=\sum_{i \geq 1}\left(P_{i}(x)+\tilde{P}_{i}(x)\right) .
$$

By Borel's theorem one can find a function $f \in C_{0}^{\infty}(\mathbb{R})$ such that its Taylor development at zero is $\hat{f}$. The inequality (27) ensures that $f \in \mathcal{U}$. Moreover observe that the property (6) implies that

$$
\forall n \geq n_{j_{1}}+1, \forall s \geq 1 \quad \mu_{n} \notin\left[u_{j_{s}}, w_{j_{s}}-1\right]
$$

and the equation (29) guarantees that, for all $n \in \mathbb{N}$,

$$
\sup _{x \in[-1,1]}\left|S_{\mu_{n}}(f)(x)\right| \leq 2 \sum_{s \geq 1} \frac{1}{(s+1)^{2}}=\frac{\pi^{2}}{3}-2 .
$$

Thus $f \notin \mathcal{U}^{(\mu)}$.

\section{ACKNOWLEDGMENTS}

The author was partly supported by the grant ANR-17-CE40-0021 of the French National Research Agency ANR (project Front).

\section{REFERENCES}

[1] F. BAyART, Boundary behavior and Cesàro means of universal Taylor series, Rev. Mat. Complut. 19 (2006), no. $1,235-247$.

[2] F. Bayart, K.-G. Grosse-Erdmann, V. Nestoridis, C. Papadimitropoulos, Abstract theory of universal series and applications, Proc. London Math. Soc. 96 (2008), 417-463.

[3] S. Charpentier, On countably universal series in the complex plane, Complex Var. Elliptic Equ. 64 (2019), no. 6, 1025-1042.

[4] G. Costakis, N. Tsirivas, Doubly universal Taylor series, J. Approx. Theory 180 (2014), 21-31.

[5] W. Gehlen, W. Luh, J. Müller, On the existence of O-universal functions, Complex Variables Theory Appl. 41 (2000), no. 1, 81-90.

[6] K-G. Grosse Erdmann, Universal families and hypercyclic operators, Bull. Amer. Math. Soc. (N.S.) 36 (1999), no. 3, 345-381.

[7] E. Katsoprinakis, Coincidence of some classes of universal functions, Rev. Mat. Complut. 22 (2009), no. 2, 427-445.

[8] W. LuH, Universal approximation properties of overconvergent power series on open sets, Analysis 6 (1986), 191-207.

[9] A. Melas, V. Nestoridis, Universality of Taylor Series as a Generic Property of Holomorphic Functions, Adv. Math. 157 (2001), 138-176.

[10] A. Mouze, On doubly universal functions, J. Approx. Theory 226 (2018), 1-13.

[11] A. Mouze, V. Munnier, Polynomial inequalities and universal Taylor series, Math. Z. 284 (2016), no. 3-4, 919-946.

[12] J. Müller, V. Vlachou, A. Yavrian, Universal overconvergence and Ostroski-gaps, Bull. London Math. Soc. 38 (2006), no. 1, 597-606.

[13] V. Nestoridis, Universal Taylor series, Ann. Inst. Fourier (Grenoble) 46 (1996), no. 5, 1293-1306.

[14] G. PÁL, Zwei kleine Bemerkungen, Tokohu Math. J. 6 (1914/15), 42-43.

[15] V. Vlachou, Disjoint universality for families of Taylor-type operators, J. Math. Anal. Appl. 448 (2017), no. 2, 1318-1330.

[16] V. Vlachou, Subclasses of universal Taylor series and center independence, arxiv:2002.03304v1 (2020).

Augustin Mouze, École Centrale de Lille, CNRS, UMr 8524 - Laboratoire Paul Painlevé 59000 Lille, France

E-mail address: augustin.mouze@univ-lille.fr 